\title{
Effects on a Poison Center's (PC) Triage and Follow-up After Implementing the No Ipecac Use Policy
}

\author{
Robert M. Lapus • Ann P. Slattery • William D. King
}

Published online: 10 July 2010

(C) American College of Medical Toxicology 2010

\begin{abstract}
For years, The American Academy of Pediatrics (AAP) had supported home use of syrup of Ipecac. However, due to mounting evidence that Ipecac use did not improve outcome nor reduce Emergency Department (ED) referrals, the AAP in November of 2003 issued a statement that Ipecac not be used for the home management of poison ingestion. To determine if the cessation of the use of Ipecac for home ingestions is associated with an increased number of follow-up calls, an increased time of observation at home and an increase in the number of ED referrals for care by poison center staff were administered. Fifty randomly selected pediatric ( $<6$ years) cases that received Ipecac ("Ipecac" group) from January 1, 2003 to October 31, 2003 were selected for study. Up to two controls ("no Ipecac" group) were matched by age, amount ingested, and by toxin. Controls were selected from the 2004-2006 time period (Ipecac no longer in use). Fifty "Ipecac" cases and 84 "no Ipecac" controls were analyzed. The groups had no significant differences with respect to percent symptomatic, median time post-ingestion, mean age, and distribution of toxin categories (e.g., antidepressants, beta blockers, etc.). The "no Ipecac" group had nearly ten times the odds of ED referral compared to the "Ipecac" group, $(\mathrm{OR}=9.9,95 \% \mathrm{CI}$ 3.3-32.2). The mean total hours of follow-up was not significantly different between the groups $(\operatorname{diff}=-1.1, t=$ $-1.8, p=0.07)$. The mean number of follow-up calls was significantly less in the "no Ipecac" group (diff $=-1.4$ calls, $t=$ $-6.8, p<0.001)$. Toxicology consults were greater in the "no Ipecac" group ( $\left.\chi^{2}=4.05, p=0.04\right)$; however, consults were not associated with ED referral. For the time period from
\end{abstract}

R. M. Lapus $(\bowtie) \cdot$ A. P. Slattery $\cdot$ W. D. King

University of Alabama,

Birmingham, AL, USA

e-mail: rlapus@peds.uab.edu
2004 to 2006, the "no Ipecac" policy resulted in an increase in ED referrals at our center. While prior studies have shown that not using Ipecac did not affect clinical outcome, our research suggested that it may have initially influenced triaging outcome. Since the use of Ipecac by centers was once a commonly used home remedy for some ingestions (albeit without rigorously established efficacy), poison center personnel had to transition to the "no Ipecac" policy. Although our referrals increased during a transitional period of time, referral rates have since stabilized and returned to baseline.

Keywords Ipecac $\cdot$ Triage $\cdot$ Poison Center .

Decontamination · Poisoning · Emergency Department . Pediatric $\cdot$ Overdose $\cdot$ Ingestion

\section{Introduction}

In October of 1965, in large part due to advocacy by The American Academy of Pediatrics (AAP), the Food and Drug Administration ruled that a 1-oz bottle of syrup of Ipecac (SOI) be available for purchase without a prescription [1] Subsequently, as part of anticipatory guidance education during visits to the pediatrician, parents were urged to have a bottle of SOI at home in the event of an accidental childhood poisoning [2]. It was strongly advocated because of the presumed ability to reduce mortality and Emergency Department (ED) referrals for the accidental ingestion of poisons. However, due to mounting evidence that SOI use did not improve outcome nor reduce ED referrals, the AAP in November of 2003 issued a statement that Ipecac no longer be used for the management of poison ingestion [3].

In the years subsequent to the policy statement change in 2003, there has been no observed significant increase in 
Table 1 Group comparability ("no Ipecac" vs. "Ipecac-given” groups)

\begin{tabular}{llll}
\hline & "no Ipecac" & "Ipecac given" & Statistics \\
\hline Median age of patient (years) & 2 & 2 & n.s. \\
Median time post-ingestion (min) & 10 & 5 & n.s. \\
Symptomatic & $3 / 81(3.6 \%)$ & $1 / 49(2 \%)$ & Log likelihood ratio $G=0.28, p=0.59$ \\
Toxicology consult & $24 / 60(28.6 \%)$ & $6 / 44(12 \%)$ & $\chi^{2}=4.1, p=0.04$ \\
& & & OR $=2.9,95 \% \mathrm{CI}(1.0-8.8)$ \\
\hline
\end{tabular}

Toxicant category was not included in the comparability analysis because toxicants were matched

morbidity or mortality associated with accidental ingestions $[4,5]$. However, not much is reported as far as what impact the policy change had on poison center triage responses. The purpose of this study was to determine if there were any statistically significant differences in triage patterns, such as the number of follow-up calls, time of observation at home, and amount of ED referrals by an urban poison center prior to and after the implementation of the "no Ipecac" statement by the AAP.

\section{Materials and Methods}

This was a retrospective case-control study of phone calls taken by the Regional Poison Control Center (RPCC) of Birmingham, Alabama. Institutional review board approval was obtained through the University of Alabama, Birmingham. We pulled the "Ipecac-given" cases from our Toxicall $^{\mathrm{TM}}$ database that were documented as "recommended and performed" under Ipecac/decontamination from January 1, 2003 through October 31, 2003 prior to the AAP November 4, 2003 Ipecac statement. We randomly selected 50 cases of children $<6$ years of age from this time period, excluding those with amounts of the substance/poison documented as unknown and those treated in a health care facility prior to calling the Poison Center. Controls for these 50 cases were retrieved from the 2004 through 2006 Toxicall TM database during which the Poison Center no longer routinely recommended Ipecac use. For each of these 50 cases, a search was done using the exact product code and matched with up to two controls by age, amount ingested, and by toxicant.

The sample size needs were determined based upon sample-derived differences in emergency department referral rates and mean total hours of follow-up between "no Ipecac" and "Ipecac-given" groups. Based upon a power of $80 \%$ and a two-tailed alpha level of 0.05 , a minimum of 41 "Ipecac-given" cases and 82 "no Ipecac" controls would be required to detect a minimum of $25 \%$ difference in emergency department referrals and a mean difference of $2 \mathrm{~h}$ total follow-up time between groups. Epi Info software provided frequency analyses to test group comparability as well as chi-square, means, and median analyses between groups. True Epistat provided Wilk's log likelihood testing and sample size analysis.

\section{Results}

A total of 50 "Ipecac-given" cases and 84 "no Ipecac" controls were included for analysis. Toxicant categories (e.g., antidepressants, anti-seizure meds, cardiovascular meds, etc.) were not included in the comparability analysis because toxicants were matched. All the cases had at least one control. The "Ipecac-given" and "no Ipecac" groups were comparable and had no statistically significant differences with respect to the percent of patients symptomatic, median time post-ingestion, mean age of the child, and whether the on-call toxicologist was called for further recommendations (see Table 1).

The "no Ipecac" group had nearly ten times the odds of ED referral compared to the "Ipecac-given" group (OR= 9.9, 95\% CI 3.3-32.2; see Table 2).

The mean total hours of follow-up was not significantly different between the "Ipecac-given" and "no Ipecac" groups (diff $=-1.1, t=-1.8, p=0.07$; see Table 3 ).

The mean number of follow-up calls was significantly less in the "no Ipecac" time period ( $\operatorname{diff}=-1.4, t=-6.8, p<0.001$; see Table 3).

Toxicology consults by the poison center staff (second level triage) were greater in the "no Ipecac" group $\left(\chi^{2}=\right.$ 4.05, $p=0.04$ ); however, consults were not associated with ED referral (see Tables 1 and 4, respectively).

Table 2 "Ipecac" groups versus ED or home triage

\begin{tabular}{lccc}
\hline & "No Ipecac" & "Ipecac given" & Totals \\
\hline ED & 41 & 5 & 46 \\
Home & 34 & 41 & 75 \\
Totals & 75 & 46 & 121 \\
$\chi^{2}=21.4, p<0.0001$ & & \\
OR $=9.995 \%$ CI $(3.3-32.2)$ & & \\
\hline
\end{tabular}

Some patients lost to follow-up or charting incomplete 
Table 3 "Ipecac" group comparisons of mean total follow-up (f/u) hours and mean total number (\#) of f/u calls

\begin{tabular}{llllll}
\hline & "No Ipecac" & "Ipecac given" & Diff & $95 \%$ CI diff & $T, p$ value \\
\hline Mean (SD) f/u hrs & $4(3.5), n=82$ & $5.1(3.1), n=48$ & -1.1 & $(-2.3,0.11)$ & $-1.8, p=0.07$ \\
Mean (SD) \# of f/u calls & $2.1(1.2), n=84$ & $3.5(1.2), n=50$ & -1.4 & $(-1.8,-0.9)$ & $-6.79, p<0.001$ \\
\hline
\end{tabular}

Some patients lost to follow-up or charting incomplete

\section{Discussion}

The landmark study by Bond published in 2003 sought to determine if use of SOI in the home was associated with reduced utilization of the ED or improved outcome. He examined the correlation between the rate of home use of SOI and the rate of ED referral. He found that increased home use of SOI was not associated with referral to the ED. Additionally, he found that when he compared two groups of poison centers divided according to "higher" vs. "lower" use of SOI, there was no significant difference between the two populations as far as referral to the ED and adverse outcomes. He concluded that the use of SOI at home did not reduce emergency department utilization nor improve outcome in the study population [3].

Our study showed that during the period examined, in patients matched by drug, age, and dose per kilogram, those in the "no Ipecac" group were nearly ten times more likely to be referred to the Emergency Department for evaluation compared to those who would have received the recommendation to be given Ipecac and be watched at home. This finding is most likely a reflection of a certain level of discomfort the poison center staff had in the aftermath of no longer being able to offer some type of intervention which had a perceived benefit, or as Vale et al. said, "To do something" [6]. This level of discomfort is also demonstrated by the increase in the RPCC calling upon the toxicologist on-call for further recommendations or reassurance with the decision to not refer the child to the ED. Our data show three interesting facts: (1) If a toxicologist was consulted for the "Ipecac-given" cases, they were also consulted for the "no Ipecac" controls. (2) If a toxicologist was consulted for the "Ipecac-given" cases, but not for their respective "no Ipecac" controls, those controls where referred to the ED for evaluation. (3) There were some toxicants for which the
"Ipecac" cases had no consults to the toxicologist; however, there were consults to the toxicologist for the "no Ipecac" controls.

This study was a retrospective chart review and therefore subject to all its inherent limitations such as selection bias and missing data or incomplete charting. Furthermore, the documentation by the RPCC staff depends upon what information the caller relays. It is conceivable that the caller may not disclose the most acurate history whether due to anxiety of the moment or out of fear that the caller may appear overly vigilant or neglectful. Inacuracies or incomplete truths by the callers in regard to dosage, drug, timing of ingestions, child weight, and prior intervention could affect our study. Other limitations in our study could be attributed to the level of experience of each RPCC staff member. More experienced members may have been able to illicit a more acurate history or may feel more comfortable with managing particular ingestions at home per set guidelines as compared to the less experienced members who may refer the child to the ED or call the toxicologist more often.

Not surprisingly, our study shows an earlier prediction to ring true, "The drug may be missed briefly but it is unlikely its absence will create more concerns that its presense has" [7]. Since the completion of this study, new triage guidelines have come into use at the RPCC. Anectdotally, we believe that should this study be repeated with the controls coming from the time period after 2007, the odds of referral would be similar, if not lower than the "Ipecac-given" time period.

\section{Conclusion}

In the period immediately following the AAP recommendation to not use SOI for accidental ingestions, calls to the

Table 4 Toxicology consults versus ED referral (to determine if toxicology consultation is associated with ED referral and thus a potential confounder to the study outcome)

\begin{tabular}{lcccc}
\hline & ED referral & Home & Percent ED referral & Statistics \\
\hline Toxicologist consulted & 10 & 18 & 35.7 & $\chi^{2}=0.004, p=0.95$ \\
Toxicologist not consulted & 36 & 58 & 38.7 & \\
\hline
\end{tabular}

Some patients lost to follow-up or charting incomplete 
RPCC regarding pediatric poisonings were associated with a significant increase in the odds of referral to the ED for evaluation. Additionally, there were also more calls by RPCC staff to the on-call toxicologist for further recommendations; however, there was no significant difference in the percent of referrals to the ED by the toxicologist. While there was an overall increase in ED referrals, there was no significant difference in the percent of patients symptomatic or admitted. The increased referrals in the period immediately after the recommendation against SOI was most certainly not due to the absence of any perceived benefit of SOI but most likely due to the fact that since the use of SOI by centers was once a commonly used home remedy for some ingestions (albeit without rigorously established efficacy), poison center personnel had to transition to the "no Ipecac" policy. Although our referrals increased during a transitional period of time, referral rates have since stabilized and returned to baseline.

\section{References}

1. Shirkey H (1966) Ipecac syrup: its use as an emetic in poison control. J Pediatr 69:139-140

2. American Academy of Pediatrics C. o. (1985) Early childhood safety counseling schedule. The injury prevention program. American Academy of Pediatrics, Elk Grove Village, IL

3. Bond G (2003) Home syrup of ipecac use does not reduce emergency department use or improve outcome. Pediatrics 112:1061-1064

4. Watson W, Litovitz T, Klein-Schwartz W, Rodgers GJ, Youniss J, Reid N et al (2004) 2003 Annual report of the American Association of Poison Control Centers Toxic Exposure Surveillance System. Am J Emerg Med 22(5):335-404

5. Watson W, Litovitz T, Rodgers GJ, Klein-Schwartz W, Reid N, Youniss J et al (2005) 2004 Annual report of the American Association of Poison Control Centers Toxic Exposure Surveillance System. Am J Emerg Med 5(23):589-666

6. Vale JA, Meredith TJ, Proudfoot AT (1986) Syrup of ipecacuanha: is it really useful? Br Med J 293:1321-1322

7. Shannon M (2003) The demise of ipecac. Pediatrics 112:1180-1181 